\title{
Glomerular filtration rate by differing measures, albuminuria and prediction of cardiovascular disease, mortality and end-stage kidney disease
}

\author{
Jennifer S Lees ${ }^{\wedge}, 1$, Claire E Welsh ${ }^{\wedge}, 1$, Carlos A Celis-Morales ${ }^{1}$, Daniel Mackay ${ }^{1}$, James \\ Lewsey ${ }^{2}$, Stuart R Gray ${ }^{1}$, Donald M Lyall ${ }^{2}$, John G Cleland ${ }^{1}$, Jason MR Gill ${ }^{1}$, Pardeep S \\ Jhund $^{1}$, Jill Pell ${ }^{2}$, Naveed Sattar ${ }^{*}, 1$, Paul Welsh ${ }^{*}, 1$, Patrick B Mark ${ }^{*}, 1$ \\ ${ }^{1}$ Institute of Cardiovascular and Medical Sciences, University of Glasgow, Glasgow, UK \\ ${ }^{2}$ Glasgow Institute of Health and Wellbeing, University of Glasgow, Glasgow, UK
}

\begin{abstract}
Chronic kidney disease is common in the general population and associated with excess cardiovascular disease (CVD), but kidney function does not feature in current CVD risk prediction models. We tested three formulae for estimated glomerular filtration rate (eGFR) to determine the most clinically informative for predicting CVD and mortality. Using data from 440,526 participants from UK Biobank, eGFR was calculated using serum creatinine, cystatin C (eGFRcys) and creatinine-cystatin C. Associations of each eGFR with CVD outcome and mortality were compared using Cox models adjusting for atherosclerotic risk factors (per relevant risk scores), and predictive utility was determined by the C-statistic and categorical Net Reclassification Index. We show that eGFRcys is most strongly associated with CVD and mortality, and along with albuminuria adds predictive discrimination to current CVD risk scores, whilst traditional creatinine-based measures are weakly associated with risk. Clinicians should consider measuring eGFRcys as part of cardiovascular risk assessment.
\end{abstract}

\footnotetext{
Users may view, print, copy, and download text and data-mine the content in such documents, for the purposes of academic research, subject always to the full Conditions of use:http://www.nature.com/authors/editorial_policies/license.html\#terms

Corresponding author: Jennifer S Lees - jennifer.lees@glasgow.ac.uk.

Joint first authors

Joint senior authors

Data availability

The UK Biobank data that support the findings of this study are available from the UK Biobank (www.ukbiobank.ac.uk). This study was conducted under project code 9310.

Author contributions

J.S.L. and P.B.M. conceived of and designed the study. Data were analysed by C.E.W. and P.W. under UK Biobank project 9310 led by N.S. and involving all authors. The first draft of the manuscript was written by J.S.L. and C.E.W. All authors (J.S.L., C.E.W., P.W., P.B.M., N.S., C.A.C-M., D.M., S.R.G., J.G.C., J.M.G., P.S.J., J.L., D.M.L., J.P.) read, critically revised and approved the final manuscript.

Competing interests

The authors declare no competing interests.
} 


\section{Introduction}

Chronic kidney disease (CKD), characterized by gradual loss of kidney function over time, is associated with progression to end-stage kidney disease (ESKD) and is an indicator for renal replacement therapy, premature cardiovascular disease (CVD) and mortality. CKD is common in the general population and affects around $10 \%$ of the total population, although estimates of its prevalence vary. ${ }^{1}$ Although impaired kidney function indicates patients at risk of future requirement of renal replacement therapy, the vast majority of patients do not require renal replacement therapy, and for these the greatest risk of CKD is the excess in cardiovascular risk associated with CKD. ${ }^{2}$ The excess CVD risk appears to begin in the relatively early stages of CKD and increases with CKD stage, especially in CKD stages G3b-5. ${ }^{2-4}$ The most pronounced effects are seen in those with advanced CKD or ESKD when the complications specific to CKD - including renal anaemia, disordered acid-base balance and CKD mineral and bone disorder - are most apparent. ${ }^{5-7}$ Though the atherosclerotic risk factors for CVD - such as hypertension, diabetes, dyslipidaemia and smoking - are prevalent in those with CKD, there is an excess CVD risk seen in CKD beyond that captured by atherosclerotic risk factors alone. ${ }^{8-12}$

Large individual patient-level meta-analyses of cohorts combining observational and clinical trial data, from the CKD Prognosis Consortium, confirm CKD as an additional, independent risk factor for CVD. ${ }^{13,14}$ Albuminuria, an important feature of CKD and glomerular damage, is thought to be associated with increased CVD risk and all-cause mortality. In additional analyses from the CKD Prognosis Consortium, albuminuria particularly improved CVD risk prediction above current methods in patients with CKD. ${ }^{15}$ As far as we are aware, only one risk calculator includes presence of CKD 3-5 as a binary risk factor for CVD risk estimation (QRISK3 ${ }^{16}$ ), whereas other modern calculators in Europe and the United States do not include CKD as a risk (Systematic Coronary Risk Evaluation (SCORE) ${ }^{17}$, American Heart Association/American College of Cardiology (AHA/ACC $)^{18}$ ).

The CKD-EPI formula ${ }^{19}$ to estimate glomerular filtration rate (GFR) has traditionally used serum creatinine values, adjusted for age, sex and ethnicity, however, in retrospective analyses, CKD-EPI formulae using cystatin $\mathrm{C}$, either alone or in combination with creatinine, perform better than those including creatinine alone in estimating $\mathrm{GFR}^{20}$ and in predicting risk of ESKD, CVD and mortality. ${ }^{21}$ Cystatin $\mathrm{C}$ testing has been available in UK National Health Service laboratories for over 10 years, and has a number of potential advantages over serum creatinine: it is released by every cell in the body, is freely filtered at the glomerulus, and is not influenced by body habitus, muscle mass or gender. It is thus thought to be a more sensitive measure to estimate kidney function. However, owing to the costs of the reagents ${ }^{22}$, cystatin $\mathrm{C}$ is around 10 times more expensive than serum creatinine at $£ 2.50$ (USD 3.00) per test compared with $£ 0.25$ (USD 0.30) for serum creatinine ${ }^{23}$. While this seems a significant additional expense, cystatin $\mathrm{C}$ costs about the same or less other standard tests conducted in patients with $\mathrm{CKD}$, including parathyroid hormone, C-reactive protein and vitamin $\mathrm{D}^{22}$. Despite being recommended by the National Institute for Health and Care Excellence (NICE) in the United Kingdom for confirmatory testing for $\mathrm{CKD}^{24}$, measurement of cystatin $\mathrm{C}$ has not been widely adopted in clinical practice, presumably relating to uncertainty around the added value of a more expensive biomarker. 
UK Biobank is one of the largest prospective population-based cohorts anywhere with extensive participant phenotyping and sampling of baseline biochemical measures including renal function (creatinine and cystatin $\mathrm{C}$ ), albuminuria (urine albumin:creatinine ratio; uACR) and lipids in around 500,000 participants. Using data from UK Biobank, we aimed to determine whether estimated GFR (eGFR) and albuminuria improves risk prediction for all-cause mortality, and CVD, using serum creatinine (eGFRcr), cystatin C (eGFRcys) and combined cystatin C-creatinine (eGFRcr-cys) estimates of GFR.

\section{Results}

\section{Demographics of participants}

Of the 502,536 participants initially included, 31,283 had missing biochemical data, 260 had prevalent ESKD, 38 had calculated eGFR (any measure) $<15 \mathrm{ml} / \mathrm{min} / 1.73 \mathrm{~m}^{2}$ and $30,112 \mathrm{had}$ prior CVD and were excluded from further analysis: therefore 440,526 participants were included in the models. Over a median of 8.9 years (Q1-Q3 8.2-9.5 years) of follow-up, 15,469 participants died from any cause, 2552 of which were deaths from CVD (based on European SCORE ${ }^{17}$ ). There were 8662 incident fatal or nonfatal CVD events (based on AHA/ACC definition ${ }^{18}$ ) and 336 cases of incident renal replacement therapy.

Participants with lower eGFR measures tended to be older, male, smokers with lower diastolic blood pressure (DBP), total and high-density lipoprotein (HDL) cholesterol, be on antihypertensive and statin medications, to report diabetes, and to be in the highest category of uACR (Table 1 and Supplementary Tables S1 and S2). eGFRcys provided lower estimates than both eGFRcr and eGFRcr-cys, resulting in a greater proportion of participants categorised as having CKD G3-5 (eGFRcr 1.9\%; eGFRcys 4.0\%; eGFRcr-cys 1.3\%).

All eGFR measures were strongly correlated, with the strongest being between eGFRcys and eGFRcr-cys $(r=0.925, \mathrm{p}<0.001)$ and weakest between the eGFRcr and eGFRcys $(\mathrm{r}=0.599$, $\mathrm{p}<0.001)$. The correlation between eGFRcr and eGFRcr-cys was $\mathrm{r}=0.828(\mathrm{p}<0.001)$.

\section{Associations between eGFR measures and all-cause mortality, CVD and ESKD outcomes}

Unadjusted survival plots for the outcomes of interest across eGFRcr categories are shown in Extended Data Figures E1-4. The adjusted shape of the associations between each eGFR measure and all-cause mortality plus both CVD outcomes and ESKD were largely linear and negative (Figure 1). This was most convincing for eGFRcys, with eGFRcr and eGFRcr-cys demonstrating greater inflections particularly at the lower extremes of eGFR. For ESKD, all three eGFR displayed an initially steep negative linear association until around $70 \mathrm{~mL} / \mathrm{min}$, and thereafter continued to decrease but at a lesser gradient (Extended Data Figure E5). Event rates per 100,000 person years for all outcomes were greater with higher eGFR by all measures (Supplementary data, Table S3).

Adjusted hazard ratios for each eGFR-outcome combination were consistent with lower eGFR being associated with higher risk of CVD, all-cause mortality, fatal CVD and ESKD (Table 2). For each outcome, there was a trend that the HR was stronger for measures of eGFR incorporating cystatin $\mathrm{C}$ for each $10 \mathrm{ml} / \mathrm{min} / 1.73 \mathrm{~m}^{2}$ increase (Table 2), which increased further alongside each 1 standard deviation increase in eGFR. The strongest 
associations (lowest HR per $1 \mathrm{SD}$ difference) were consistently found for eGFRcys (HRs $0.72,0.80,0.66,0.14$, for all-cause mortality, composite CVD, fatal CVD and ESKD/renal replacement therapy outcomes, respectively, Table 2).

\section{Prediction of all-cause mortality and CVD with albuminuria}

There was an association between decreasing eGFR and increased hazard ratio for all outcomes that was sustained across uACR groups, though the magnitude of the association was similar in higher compared with lower uACR groups (Figure 2 and Supplementary data Table S4). As expected, the risk of ESKD increases with higher uACR category (Figure 2 and Supplementary data Table S4). Addition of uACR to atherosclerotic risk factors and eGFR was associated with mortality and CVD across the full cohort (Supplementary data Tables S5-S7). Figure 2 shows heat maps for prediction of all-cause mortality and CVD events using AHA/ACC and SCORE criteria using eGFRcys and uACR groups. Addition of albuminuria to eGFRcys and atherosclerotic risk factors did not improve Net Reclassification Index across $7.5 \% 10$ year risk threshold used in AHA/ACC guidelines ${ }^{18}$ (Table 3).

\section{Prediction of all-cause mortality and CVD outcomes by eGFR measures}

For all-cause mortality, atherosclerotic risk factors (age, sex, ethnicity, systolic blood pressure, diastolic blood pressure, antihypertensive medications, statin use, smoking, diabetes, total and HDL cholesterol) yielded a C-statistic of 0.7157 (95\% CI 0.7115-0.7200), Figure 3). Addition of both eGFRcys and eGFRcr-cys significantly improved discrimination, with the largest improvement seen with eGFRcys (C-statistic $+0.0103,95 \%$ CI 0.0087-0.0121). Similarly, for the composite fatal/non-fatal CVD outcome (based on AHA/ACC risk score), atherosclerotic risk factors yielded a C-statistic of 0.7387 (95\% CI $0.7337-0.7439$ ) which was improved by addition of eGFRcys (C-statistic $+0.0039,95 \%$ CI 0.0025-0.0052). Similarly, and for fatal CVD (based on SCORE), atherosclerotic risk factors (C-statistic 0.7828 , 95\% CI 0.7740-0.7917) were improved by addition of the eGFRcys (Cstatistic change $+0.0085(0.0049-0.0122)$.

For all-cause mortality, and both CVD outcomes, addition of eGFRcr did not improve discrimination. For the CVD outcome, we tested improvement in risk classification across the $7.5 \% 10$ year risk threshold for statin therapy used in AHA/ACC guidelines. ${ }^{18}$ eGFRcr did not improve risk classification, but measures incorporating eGFRcys did (Table 3).

\section{Subgroup analyses: prediction of all-cause mortality and CVD}

Across all subgroups, eGFRcr did not improve prediction of all-cause mortality or either CVD outcome in addition to atherosclerotic risk factors. eGFRcys improved prediction of all-cause mortality in all subgroups (Supplementary Table S5), and prediction of at least one CVD outcome in subgroups except non-white ethnicity and CKD3b/4 (Supplementary Tables S6 and S7). In a model incorporating atherosclerotic risk factors and uACR, eGFRcys improved prediction of mortality across all subgroups (Supplementary Table S5); eGFRcys improved prediction of at least one CVD outcome in subgroups except non-white ethnicity, CKD 3b/4 and BMI 30-35 kg/m² (Supplementary Tables S6 and S7). As a sensitivity analysis for data linearity, the performance of each eGFR was tested for all outcomes in 
participants with eGFRcr $<60 \mathrm{ml} / \mathrm{min} / 1.73 \mathrm{~m}^{2}$ with consistent conclusions: eGFRcr did not improve outcome prediction for all-cause mortality or either CVD outcome; addition of eGFRcys most strongly improved model prediction across all 4 outcomes (Supplementary Table S8). Across all models in and in every subgroup, the likelihood ratio test comparing addition of uACR to models containing atherosclerotic risk factors and eGFR produced $\mathrm{p}$ value $<0.001$.

\section{Discordance analysis}

There was absolute discordance $\geq 20 \%$ between eGFRcr and eGFRcys measurements in 183,867 participants (41.47\%). Baseline characteristics were broadly similar amongst those with discordant compared with concordant eGFRcr and eGFRcys (Supplementary data Table S9). Amongst those with discordant results, eGFRcys remained the marker with the greatest improvement in C-statistic, adjusted for atherosclerotic risk factors and uACR, across mortality and both CVD outcome measures (Table 4). None of the eGFR measures improved prediction over atherosclerotic risk factors and UACR for ESKD (Table 4), though there were very few ESKD events $(n=52)$ in this subgroup and the impact of eGFR in this discordant group may be under-estimated.

\section{Discussion}

This is the largest prospective cohort study to demonstrate additional reclassification of cardiovascular risk using eGFRcys, with no added predictive value of traditional eGFRcr. We also demonstrate eGFRcys to be more strongly associated with future CVD events than eGFRcr for both CVD outcomes, and this message is consistent across most subgroup analyses. It is not surprising that baseline kidney function and albuminuria are associated with increased risk of ESKD. In this cohort, broadly representative of the general UK population, eGFRcys was more closely associated with this outcome than eGFRcr - the measure used in current clinical practice.

Estimation of renal function from creatinine has become more reliable since creatinine measurements were standardised to IDMS-traceable techniques ${ }^{25}$, however, creatinine remains an imperfect tool to estimate GFR. Released from the breakdown of muscle tissue, creatinine will be more abundant in those with extremes of muscle mass, body habitus or dietary habits. eGFRcr will therefore tend to overestimate GFR (and underestimate presence or severity of CKD) in older individuals or those with less than average muscle mass for their age and is an insensitive marker of kidney impairment in early disease. Cystatin $\mathrm{C}$ is a small protein produced by all nucleated cells (so is less susceptible to influence by individual patient characteristics) and is freely filtered at the glomerulus. Cystatin $\mathrm{C}$ is thought to be a more sensitive blood marker of kidney function than is creatinine, and is not influenced by muscle mass, age, gender or ethnicity.

Cystatin C, however, is correlated with oxidative stress and inflammation ${ }^{26,27}$. Various cardiometabolic conditions are associated with higher levels of cystatin C; we have adjusted for some within the current risk prediction tools (diabetes, dyslipidaemia, hypertension). Patients with other diagnosed or undiagnosed cardiometabolic conditions, such as thyroid disease, cancer and glucocorticoid therapy, may have slightly higher levels of cystatin C, 
confounding the observed effect of lower eGFRcys on outcome. This may also explain the difference we observed in the strong aetiological association between eGFRcys and outcome with modest improvement in risk prediction ${ }^{28}$. Observed effects of lower eGFR on cardiovascular risk may be mediated by inadequate adjustment for atherosclerotic risk factors in patients with CKD in current risk prediction models. The contribution of nontraditional CVD risk factors in those with moderate CKD may be less pronounced than previously thought and use of eGFRcys may serve as a biomarker that better captures risk associated with other cardiometabolic conditions.

Amongst patients with CKD, CVD risk-reduction relies on three important factors: 1) accurate diagnosis of CKD; 2) recognition of the elevated risk of CVD; and 3) early identification and treatment of modifiable risk factors.

In the UK, NICE guidelines recommend considering a cystatin C-based calculator to confirm or refute a diagnosis of $\mathrm{CKD}^{24}$. This recommendation is not being undertaken in routine clinical practice and the diagnostic and prognostic utility of cystatin C in CKD stage G3 is currently being tested in a prospective study. ${ }^{29}$ We confirm the observation that reduced kidney function and albuminuria indicate groups at extremely high risk of need for future renal replacement therapy, as outlined in the Kidney Failure Risk Equation. ${ }^{30}$ Our data show that eGFRcys identified a greater proportion of participants with CKD G3-5 than both eGFRcr and eGFRcr-cys, and is more strongly associated with clinical outcome than either of the other measures. On this basis, we suggest that eGFRcys should be used for diagnosis of CKD. This could apply both for confirmation of CKD in patients with eGFRcr $<60 \mathrm{ml} / \mathrm{min} / 1.73 \mathrm{~m}^{2}$ or for screening for CKD in patients with CVD risk factors, such as diabetes, hypertension or obesity.

A more accurate diagnosis of CKD should then prompt assessment and treatment of modifiable risk factors including diabetes mellitus, hypertension, smoking and dyslipidaemia. The efficacy of statins in reducing atherosclerotic CVD effects has been demonstrated in randomised clinical trials ${ }^{31,32}$. However, the effectiveness of statins in reducing risk of CVD in patients with CKD reduces as eGFR declines, partly as the mode of CVD events in advanced CKD becomes less driven by atherosclerotic effects and more by heart failure and sudden cardiovascular death. Therefore, cholesterol-lowering treatments are recommended for primary prevention in all patients with non-dialysis dependent CKD who are over 50 years or any patient with CKD and diabetes ${ }^{33}$. Similar arguments pertain to the treatment of blood pressure, which is a risk factor for both future CVD and CKD progression. Accurate documentation of kidney function should prompt consideration of whether to aim for lower blood pressure targets as informed by the SPRINT trial and its CKD subgroup analysis. ${ }^{34,35}$ Whilst the added NRI of $1.54 \%$ for CVD in this study seems modest, $1.37 \%$ more cases were appropriately identified which, when multiplied by how many people receive CVD risk scoring, would amount to a large number of people worldwide. Indeed, in participants in the UK Biobank, addition of eGFRcys to atherosclerotic risk factors improves prediction and reclassification of CVD more substantially than does addition of total and HDL-C cholesterol to non-lipid atherosclerotic risk factors ${ }^{36}$. Our results - showing eGFRcys as the most appropriate measure of renal 
function and predicting cardiovascular diseases - are therefore clinically important. eGFRcys should be incorporated into cardiovascular risk prediction tools.

Supporting published data from other large cohort studies ${ }^{13,14,37}$, we have shown that eGFR is independently associated with CVD events and mortality, and CVD event rates were augmented as eGFR declined ${ }^{3}$. Our conclusions assume a linear relationship between eGFR measures and outcome, in keeping with other published analyses ${ }^{14,21,37}$. Both eGFRcys and eGFRcr demonstrated some increase in CVD, mortality and ESKD risk below $90 \mathrm{ml} / \mathrm{min} /$ $1.73 \mathrm{~m}^{2}$, however, there was pronounced flattening of the risk association across all outcomes for eGFRcr $\sim 75-90 \mathrm{ml} / \mathrm{min} / 1.73 \mathrm{~m}^{2}$. eGFRcys demonstrated a much stronger, linear association with risk of mortality, CVD and ESKD below eGFRcys $90 \mathrm{ml} / \mathrm{min} / 1.73 \mathrm{~m}^{2}$. Within the group with eGFRcys $60-89 \mathrm{ml} / \mathrm{min} / 1.73 \mathrm{~m}^{2}$, HRs for all-cause mortality and both cardiovascular outcomes are mostly $\sim 1.3$ or higher, compared with HRs close to the reference for eGFRcr at the same level. eGFRcys is therefore better suited for early detection of increased risk of these outcomes, both through its strong linear association, and a tendency to estimate lower GFR (and therefore higher risk). Within our population, 37.5\% had eGFRcr $60-89$, increasing to $46.3 \%$ with eGFRcys. This represents a substantial group of patients who could benefit from the added predictive value of eGFRcys - at relatively low cost of $£ 2.50$ (USD 3.00) per test - particularly if used judiciously at point of CKD diagnosis or for one-off use in cardiovascular risk prediction tools. The UK Biobank population may not be representative of the UK population with CKD G3-5 prevalence $<2.0 \%$ by eGFRcr or $\sim 4 \%$ by eGFRcys; across England, the prevalence of CKD G3-5 in adults is around $6.1 \% 38$. Applied to a real-life population with a greater burden of kidney disease, the potential benefit of measuring eGFRcys for risk prediction may be augmented.

In keeping with previous analyses from the Chronic Kidney Disease Prognosis Consortium (combining administrative datasets, observational cohorts and clinical trials) $)^{13,14,37}$, we have found albuminuria to be associated with mortality and cardiovascular events. Our hazard ratios for all-cause mortality and fatal cardiovascular disease were broadly similar across uACR groups and eGFR categories to those found in a previous analysis from the Chronic Kidney Disease Prognosis Consortium ${ }^{14}$, though we report lower hazard ratios for fatal CVD for those with eGFR $>45$ and $\mathrm{uACR}>30 \mathrm{mg} / \mathrm{mmol}$, but higher HR for those with eGFR $<45$ and $\mathrm{uACR}>30 \mathrm{mg} / \mathrm{mmol}$ than reported previously ${ }^{14}$. In contrast to previous studies, we did not find albuminuria to improve risk prediction over atherosclerotic risk factors and eGFRcys in reclassification models. In the UK Biobank, only 3.2\% of 440,526 participants had uACR $>30 \mathrm{mg} / \mathrm{mmol}$, compared to $11.5 \%$ of 105,715 participants representing the general population in the Chronic Kidney Disease Prognosis Consortium ${ }^{14}$. Furthermore, albuminuria was estimated from random spot urine samples, though early morning samples have been shown to correlate more closely with 24-hour urinary albumin concentration ${ }^{39}$. The risk associated with heavy albuminuria in our study may have been underestimated.

The strengths of this study lie in the large scale, prospective population-based cohort containing complete cases and a low proportion $(0.06 \%)$ of excluded cases due to missing data. The outcomes are obtained from linked health records and through self-reporting measures and therefore are likely to have captured the majority of hard endpoints of interest $\left(\mathrm{CVD}\right.$ and death $\left.{ }^{40}\right)$. The data were obtained from a single-protocol study conducted in 
accordance with published protocols and measurement of biochemical data was centralised, affording consistent measurement of creatinine and cystatin $\mathrm{C}$ values used in the GFR calculators across all participants ${ }^{40}$.

We acknowledge some weaknesses in the data presented. Though the population studied was broadly representative of a real-life cohort (i.e. many had comorbidities including diabetes mellitus, hypertension and other comorbid disease), included participants were volunteers for the UK Biobank resource and therefore may not be representative of the breadth of comorbidity seen in the general population, ${ }^{41}$ particularly given the $\sim 5 \%$ response rate. Reflecting the general population, there was a relatively small proportion with advanced CKD (stages G4/5) and relatively few ESKD events. eGFR measures may perform differently in later stage CKD, particularly with reference to prediction of ESKD, and this subgroup warrants more specific study. Renal outcomes were identified from linked health records, but are more reliably obtained from designated renal resources such as the UK Renal Registry; ${ }^{42}$ renal outcomes were not linked to national registries and some ESKD outcomes may have been missed. Similarly, cardiovascular endpoints obtained from linked health records will have captured most events, but these events have not been validated with the same rigour as in dedicated cardiovascular studies, and some cardiovascular endpoints may have been missed or incorrectly coded. The ethnic groups were representative of a UK population with a bias towards white ethnic groups. We did not find eGFRcys to be predictive of CVD outcome in non-white ethnic groups, though with limited non-white participants it is difficult to draw any firm conclusions in other ethnic groups. We have not adjusted for all cardiometabolic conditions associated with higher levels of cystatin $\mathrm{C}$ (including cancer, glucocorticoid therapy or thyroid disease), nor were participants with these conditions excluded. Inclusion of participants with these conditions may have overestimated the impact of eGFRcys on outcome. Finally, GFR has been estimated from serum biomarkers and not measured using radioisotope studies, iohexol clearance or formal inulin clearance studies. Nevertheless, estimation of GFR is standard practice in the diagnosis of CKD. That noted, the size and scope of UK Biobank means that our data particularly on CVD risk with eGFR data add strongly to this literature on this important question.

In summary, we have shown that cystatin C-based calculations of GFR provide for more accurate prediction of all-cause mortality and fatal/non-fatal CVD. eGFRcys and albuminuria are independent risk factors for fatal/non-fatal CVD and should be considered in cardiovascular risk prediction to advise primary preventative treatment decisions. Consideration should be given to measuring serum cystatin $\mathrm{C}$ and using eGFRcys for diagnosis of CKD, prediction of CVD and thus making important clinical decisions around implementation of CVD risk lowering therapies in addition to conventional CVD risk factor calculators.

\section{Methods}

UK Biobank collected data from 502,536 consenting participants (age 37 to 73) from 2007-2010 across 22 assessment centres in the UK. Biological data and information from touch-screen questionnaires were collected at baseline as previously described. ${ }^{43,44}$ Ethical 
approval for UK Biobank was issued by the North West Multi-centre Research Ethics Committee (REC reference: 11/NW/03820). The study was conducted in accordance with the principles of the Declaration of Helsinki, and all participants gave written informed consent before enrolment.

Ethnicity was initially coded as white, black, south Asian or other, but for the purposes of eGFR calculators, ethnicity was coded as black or other. Smoking history was self-reported and categorised as current/previous or never smoker. We excluded those with prevalent ESKD or who were receiving renal replacement therapy in any form at baseline, defined from self-reported ESKD according to a pre-specified algorithm. ${ }^{45}$ We further excluded any participant with a calculated eGFR from any measure of $<15 \mathrm{ml} / \mathrm{min} / 1.73 \mathrm{~m}^{2}$. Participants with previous history of CVD (self-reported angina, myocardial infarction, stroke or transient ischaemic attack) were excluded.

Full details of the biochemistry sampling, handling and quality control protocol for UK Biobank has been described and validated previously. ${ }^{40,46-48}$ In brief, blood and spot urine samples were collected and analysed at a central laboratory, including creatinine, cystatin C, lipids (high-density, low-density and total cholesterol) and urine albumin content (urine albumin:creatinine ratio; uACR). The UK Biobank operated a high-turnover, clinic setup and thus samples were collected at various times of day. Serum and urine creatinine were measured using an enzymatic (creatinase), IDMS-traceable, method on Beckman Coulter AU5400 instrument. ${ }^{49}$ Serum cystatin C was measured by latex enhanced immunoturbidimetric method on a Siemens ADVIA 1800 instrument. ${ }^{49}$ Urine microalbumin was measured by immunoturbidimetric method using reagents and calibrators sourced from Randox Bioscience (UK) ${ }^{50}$. Over 3 levels of control the coefficient of variation for creatinine was $<2.8 \% 48$, for cystatin $\mathrm{C}$, over 2 levels of control, was $<1.4 \% 48$ and for urinary microalbumin and creatinine, over 2 levels of control, was $<2.1 \%{ }^{50}$. Each assay was registered with an external quality assurance (EQA) scheme, and assay performance was externally verified via the results returned from participation in these schemes. Data were adjusted by UK Biobank centrally before release to adjust for preanalytical variables ${ }^{51}$. Estimated glomerular filtration rate was calculated by CKD-EPI using serum creatinine (eGFRcr) ${ }^{19}$, cystatin C (eGFRcys) or cystatin C-creatinine (eGFRcr-cys) equations as previously reported. ${ }^{20}$

There were four outcomes of interest. First, all-cause mortality was defined as death from any cause, with date and cause of death obtained from death certificates held by the National Health Service (NHS) Information Centre (participants in England and Wales) or the NHS Central Register Scotland (for participants from Scotland). Second, composite fatal or nonfatal cardiovascular disease (CVD) events (nonfatal episodes of MI, stroke, or heart failure ICD10 codes I22, I24, I60, I61, I63 or I64, or fatal CVD ICD10 codes I20-I25, I60-I64) were identified by linkage with routine hospital data, and date and cause of death (where appropriate) were obtained from death certificates as for all-cause mortality. Third, fatal CVD events were identified from fatal CVD ICD10 codes (I20-I25, I60-I64) and from death certificates as for all-cause mortality. Last, ESKD was defined as reaching CKD stage G5 or requirement for renal replacement therapy, using hospital admission ICD10 (E85.3, N16.5, N18.0, N18.5, Q60.1, T82.4, T86.1, Y60.2, Y61.2, Y62.2, Y84.1, Z49.0, Z49.1, Z49.2, 
Z94.0, Z99.2) and OPCS4 (L74.1, L74.2, L74.3, L74.4, L74.5, L74.6, L74.8, L74.9, M01.2, M01.3, M01.4, M01.5, M01.8, M01.9, M02.3, M08.4, M17.2, M17.4, M17.8, M17.9, $\mathrm{X} 40.1, \mathrm{X} 40.2, \mathrm{X} 40.3, \mathrm{X} 40.4, \mathrm{X} 40.5, \mathrm{X} 40.6, \mathrm{X} 40.7, \mathrm{X} 40.8, \mathrm{X} 40.9, \mathrm{X} 41.1, \mathrm{X} 41.2, \mathrm{X} 41.8$, $\mathrm{X} 41.9, \mathrm{X} 42.1, \mathrm{X} 42.8, \mathrm{X} 42.9, \mathrm{X} 43.1)$ codes, or ICD10 codes (N18.0, N18.5) listed in any position in a death record, according to a pre-specified algorithm. ${ }^{45}$.

The follow-up period started at the date of first assessment. The follow-up period ended with the date of death, first date of hospitalisation for non-fatal CVD or ESKD, or end of followup (whichever occurred first). For mortality endpoints, end of follow-up was recorded as the first of date of death, or the end of data collection for the attended assessment centre (30/11/2016 for centres in Scotland; 31/1/2018 for centres in England/Wales).

\section{Statistical analysis}

Each eGFR was categorised into the following five groups $\left(\mathrm{ml} / \mathrm{min} / 1.73 \mathrm{~m}^{2}\right)$ aligned with KDIGO staging of chronic kidney disease: $\geq 90,60-89,45-59,30-44,15-30 .{ }^{11}$ The distributions of atherosclerotic risk factors (age, sex, ethnicity, smoking, systolic and diastolic blood pressure, use of antihypertensive medication and statins, total and HDL cholesterol) and uACR were investigated across eGFR categories and across each outcome. Continuous risk factors were displayed as mean (SD) if normally distributed and median (Q1-Q3) if skewed. Categorical risk factors were displayed as count (\%). Tests for trends across categories were performed using chi-squared tests, ANOVA or Wilcoxon rank-sum tests where appropriate.

The event rate per 100,000 person years for each outcome in each category of eGFR was calculated using the Kaplan-Meier method, and the event-free survival rate per category was plotted using this method.

Pearson correlation coefficients between the three linear eGFR measures were calculated. To examine the relationship between each eGFR-outcome combination, restricted cubic splines (with knots at eGFR of 20, 30, 45, 60, 75, 90, 105 and $115 \mathrm{ml} / \mathrm{min} / 1.73^{2}$ ) were constructed and each fully adjusted relationship (adjusted for age, sex, ethnicity, smoking, systolic and diastolic blood pressure, antihypertensive medications, statins, total and HDL cholesterol) was plotted.

To assess the effect of the addition of each eGFR measure and albuminuria to the discriminative ability of atherosclerotic risk factors, Cox-proportional hazard models (adjusted as above) were constructed for all-cause mortality, fatal/non-fatal CVD or fatal CVD, and the change in model discrimination was assessed using Harrell's C-statistics for each eGFR, in isolation and with addition of albuminuria. To understand better the relationship between eGFR and each outcome in the context of different uACR levels, uACR was split into clinically meaningful groups ( $\mathrm{UACR}<3,3-30$ or $>30 \mathrm{mg} / \mathrm{mmol}$ according to the KDIGO clinical practice guideline for definition and classification of $\mathrm{CKD}^{11}$ ) and the hazard ratio for each category of eGFR within each uACR group was assessed.

eGFR and albuminuria were modelled linearly, with log transformation for uACR. Similar analyses were conducted in subgroups including gender, ethnicity (white vs. non-white), 
body mass index $\left(<30,30-35,>35 \mathrm{~kg} / \mathrm{m}^{2}\right)$ and CKD 3b/4 (eGFRcr $15-45 \mathrm{ml} / \mathrm{min} / 1.73 \mathrm{~m}^{2}$ ). As a sensitivity analysis, $\mathrm{C}$-statistics for change in model discrimination for each outcome in the group of participants with eGFRcr $<60 \mathrm{ml} / \mathrm{min} / 1.73 \mathrm{~m}^{2}$, to check the influence of data linearity above $60 \mathrm{ml} / \mathrm{min} / 1.73 \mathrm{~m}^{2}$, Categorical Net Reclassification Index was tested for reclassification of patients from "low" to "intermediate" risk, i.e. across the threshold 7.5\% 10 -year risk of CVD that would warrant initiation of statin therapy for CVD risk reduction. 18

As a sensitivity analysis we assessed cases in which there was $\geq 20 \%$ absolute discordance between eGFRcr and eGFRcys. Adjusted Cox-proportional hazard models (as above) were repeated for the group in which there was $>20 \%$ discordance for all 4 outcomes.

We excluded participants with missing data and who reported baseline CVD or ESKD, or who had eGFR (any measure) $<15 \mathrm{ml} / \mathrm{min} / 1.72 \mathrm{~m}^{2}$; all analyses were performed on complete cases. Analyses were performed using STATA 14 (StataCorp LP, College Station, USA) and nricens for R statistical software package (version 3.5.3) for NRI. A p-value of $<0.05$ was considered statistically significant.

\section{Extended Data}




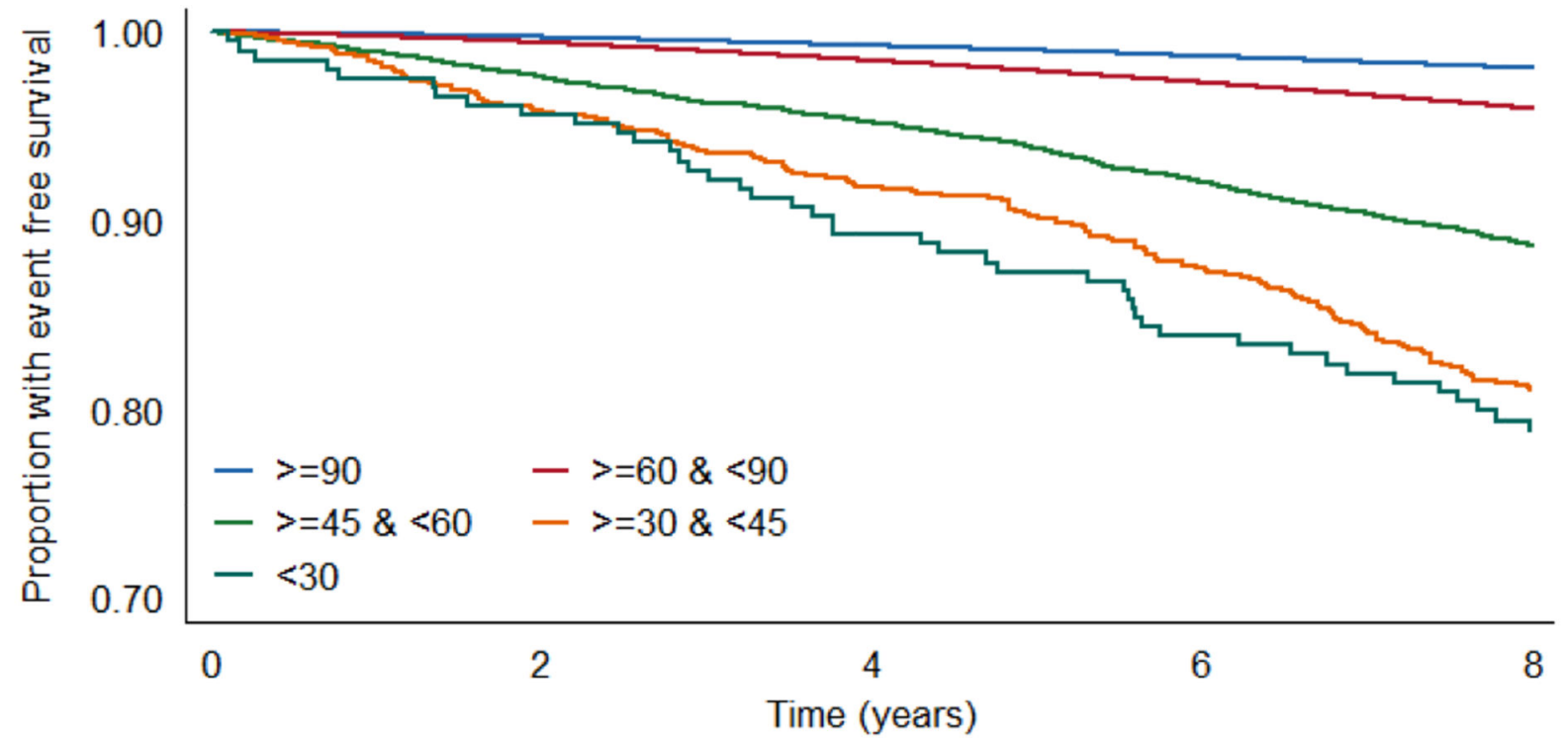

Number at risk

\begin{tabular}{|c|c|c|c|c|}
\hline $90+247892$ & 247248 & 246135 & 244733 & 206425 \\
\hline $60-90186928$ & 185905 & 184139 & 181956 & 151134 \\
\hline $45-60 \quad 4706$ & 4595 & 4483 & 4334 & 3489 \\
\hline $30-45 \quad 832$ & 798 & 764 & 728 & 562 \\
\hline$<30 \quad 205$ & 196 & 183 & 172 & 140 \\
\hline
\end{tabular}

Extended Data Figure E1. 


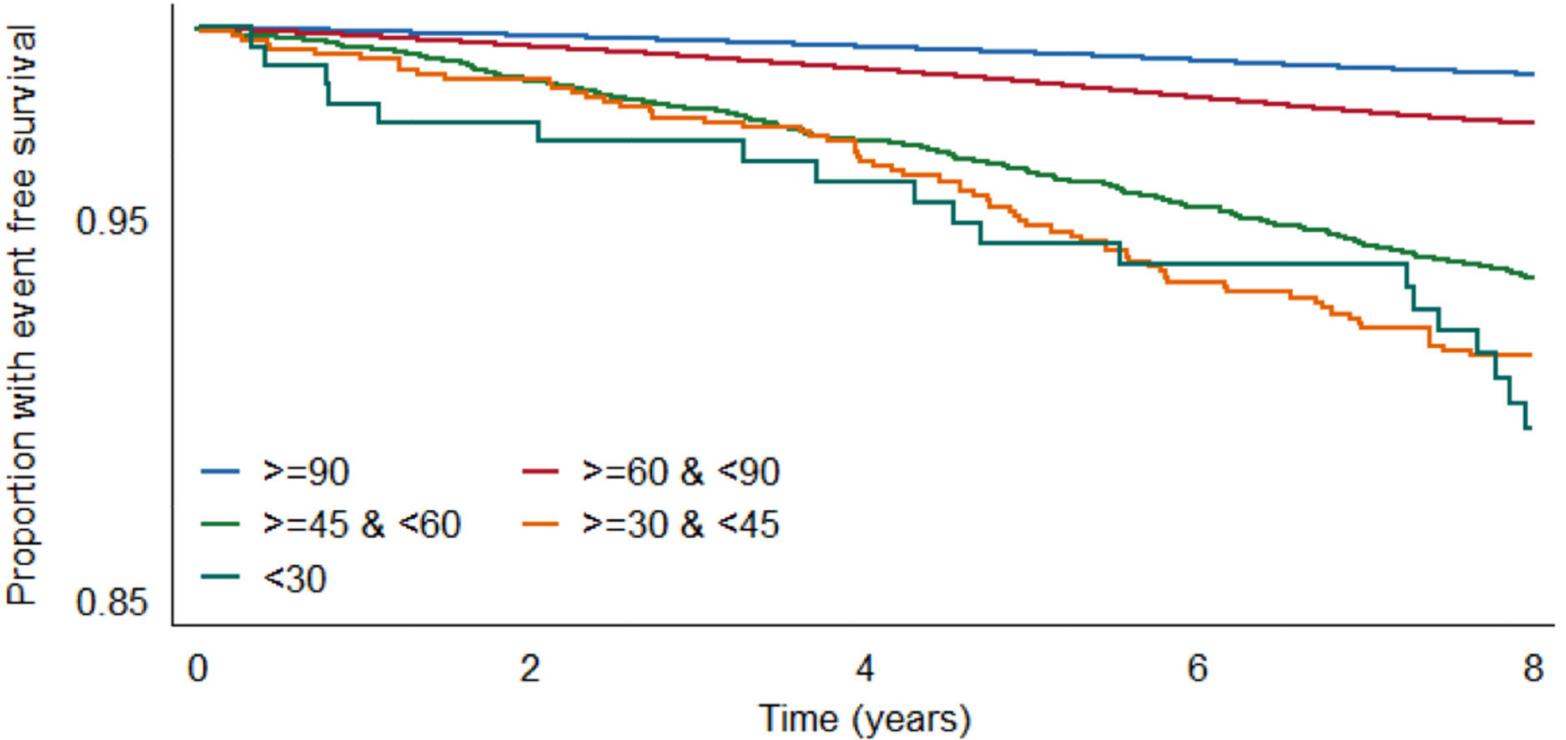

Number at risk

\begin{tabular}{|c|c|c|c|c|}
\hline $90+247892$ & 246818 & 245136 & 243052 & 204360 \\
\hline $60-90186928$ & 185168 & 182541 & 179323 & 148161 \\
\hline $45-60 \quad 4706$ & 4558 & 4395 & 4203 & 3329 \\
\hline $30-45 \quad 832$ & 792 & 747 & 694 & 534 \\
\hline$<30 \quad 205$ & 192 & 179 & 167 & 132 \\
\hline
\end{tabular}

Extended Data Figure E2. 


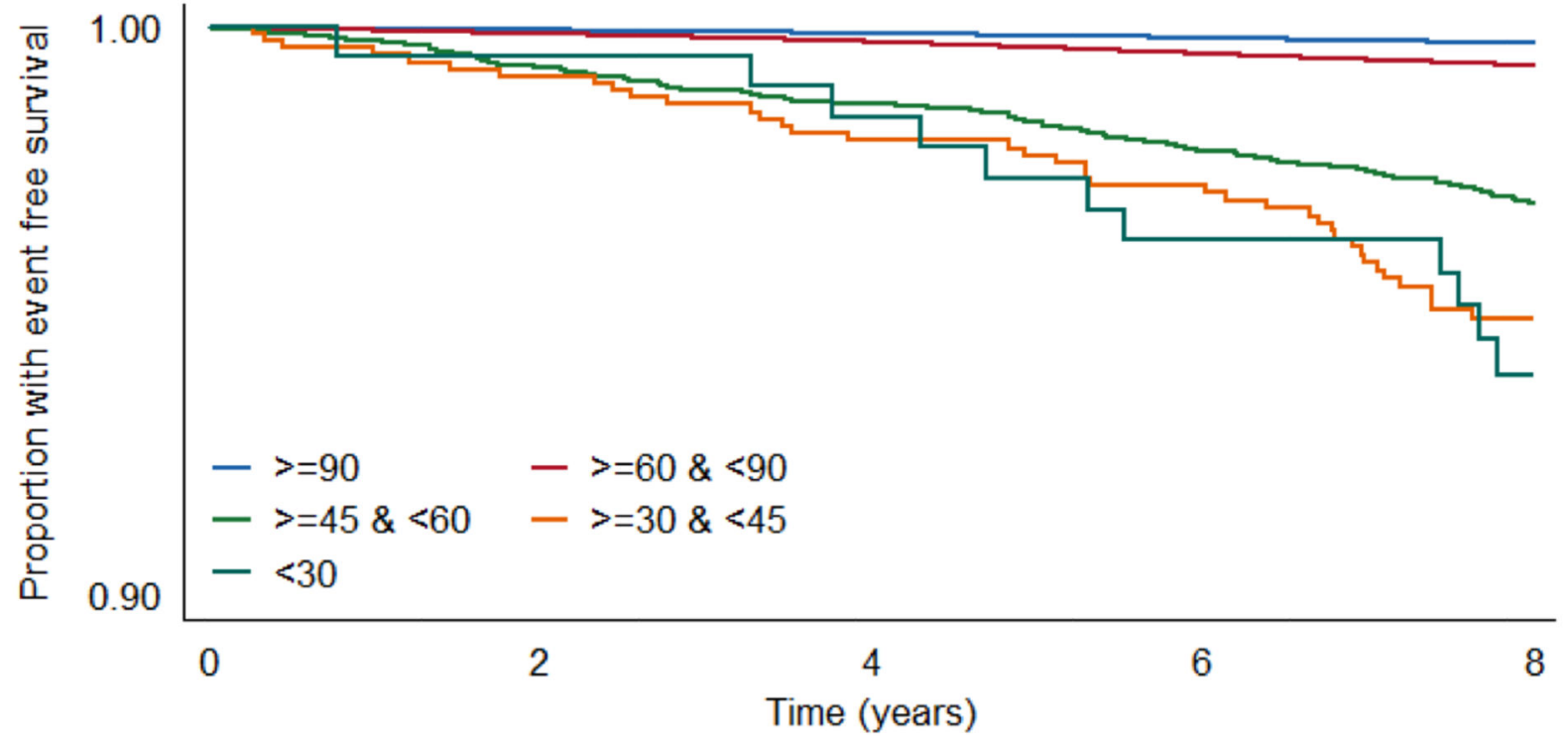

Number at risk

\begin{tabular}{|c|c|}
\hline $90+247892$ & 247248 \\
\hline $60-90186928$ & 185905 \\
\hline $45-60 \quad 4706$ & 4595 \\
\hline $30-45$ & 798 \\
\hline$<30 \quad 205$ & 196 \\
\hline
\end{tabular}

246135
184139
4483
764
183

Extended Data Figure E3. 


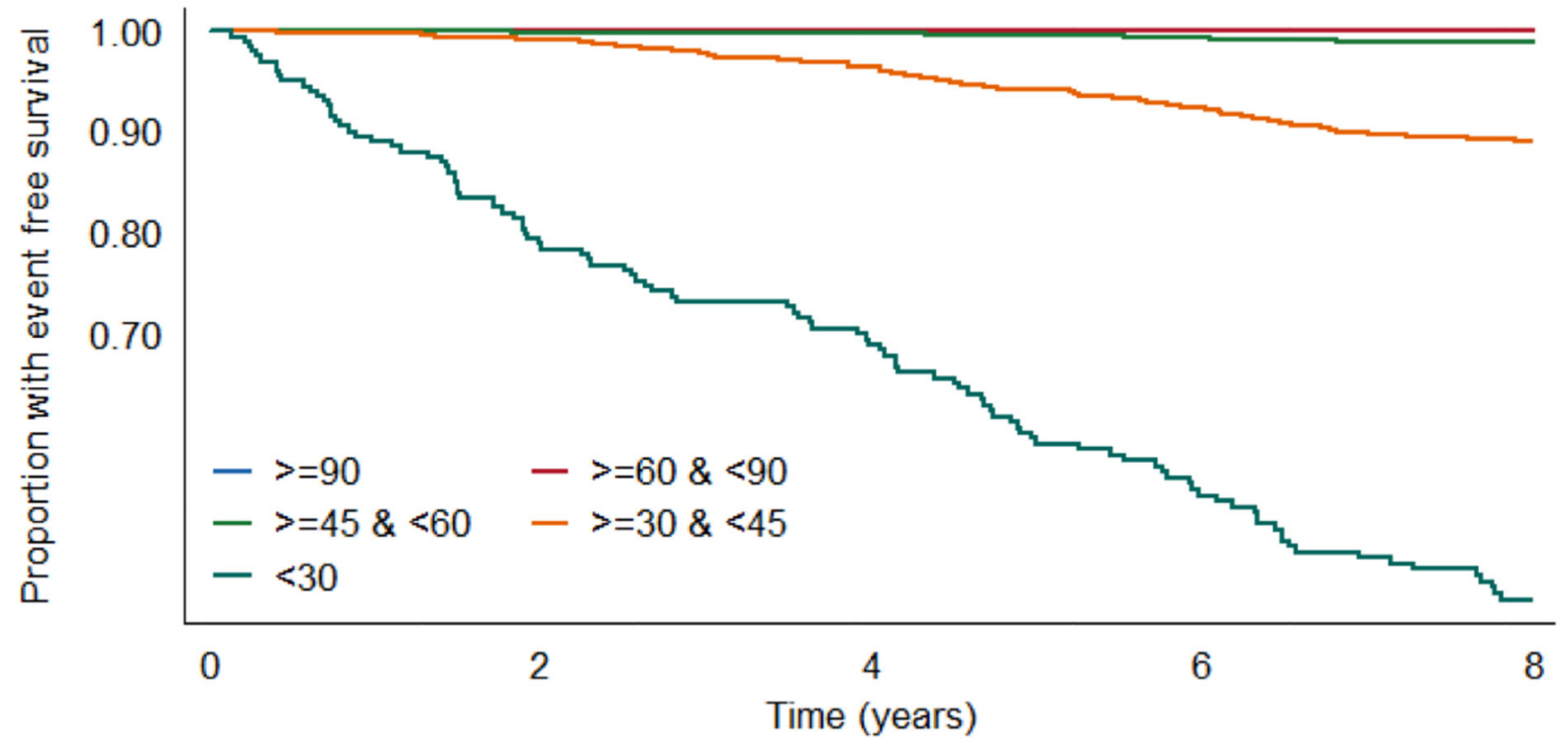

Number at risk

$\begin{array}{rc}90+247892 \\ 60-90 & 186928 \\ 45-60 & 4706 \\ 30-45 & 832 \\ <30 & 205\end{array}$

247245
185898
4591
791
154

246124
184127
4473
737
128

Extended Data Figure E4. 

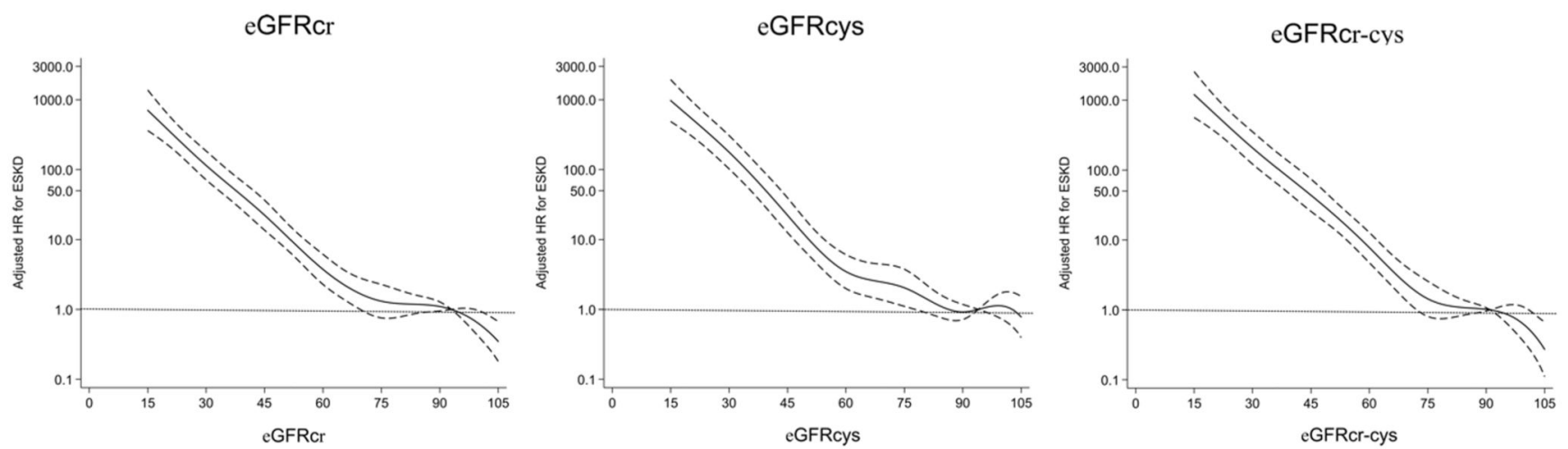

Extended Data Figure E5.

\section{Supplementary Material}

Refer to Web version on PubMed Central for supplementary material.

\section{Acknowledgements}

We would like to thank the participants of the UK Biobank. The work in this study was supported by a grant from Chest, Heart and Stroke Association Scotland (Res16/A165). J.S.L. has personal funding from a Kidney Research UK Training Fellowship Award (TF_013_20161125) and is supported by a British Heart Foundation Centre of Excellence Award (RE/13/5/30177).

\section{References}

1. Hill NR, Fatoba ST, Oke JL, Hirst JA, O’Callaghan C, et al. Global Prevalence of Chronic Kidney Disease - A Systematic Review and Meta-Analysis. PLoS One. 2016; 11(7):e0158765. [PubMed: 27383068]

2. Evans M, Grams ME, Sang Y, Astor BC, Blankestijn PJ, et al. Risk Factors for Prognosis in Patients With Severely Decreased GFR. Kidney Int Reports. 2018; 3:625-637.

3. Go AS, Chertow GM, Fan D, McCulloch CE, Hsu C. Chronic Kidney Disease and the Risks of Death, Cardiovascular Events, and Hospitalization. N Engl J Med. 2004; 351:1296-1305. [PubMed: 15385656]

4. Grams ME, Sang Y, Ballew SH, Carrero JJ, Djurdjev O, et al. Predicting timing of clinical outcomes in patients with chronic kidney disease and severely decreased glomerular filtration rate. Kidney Int. 2018; 93:1442-1451. [PubMed: 29605094]

5. Stauffer ME, Fan T. Prevalence of anemia in chronic kidney disease in the United States. Public Libr Sci one. 2014; 9

6. Neri L, Kreuzberg U, Bellocchio F, Brancaccio D, Barbieri C, et al. Detecting high-risk chronic kidney disease-mineral bone disorder phenotypes among patients on dialysis: a historical cohort study. Nephrol Dial Transplant. 2019; 34:682-691. [PubMed: 30165528]

7. Levin A, Bakris GL, Molitch M, Smulders M, Tian J, et al. Prevalence of abnormal serum vitamin D, PTH, calcium, and phosphorus in patients with chronic kidney disease: Results of the study to evaluate early kidney disease. Kidney Int. 2007; 71:31-38. [PubMed: 17091124]

8. Sarnak MJ, Levey AS, Schoolwerth AC, Coresh J, Culleton B, et al. Kidney Disease as a Risk Factor for Development of Cardiovascular Disease: A Statement From the American Heart Association Councils on Kidney in Cardiovascular Disease, High Blood Pressure Research, Clinical Cardiology, and Epidemiology and Prevention. Circulation. 2003; 108:2154-2169. [PubMed: 14581387] 
9. Israni AK, Snyder JJ, Skeans MA, Peng Y, MacLean JR, et al. Predicting coronary heart disease after kidney transplantation: Patient Outcomes in Renal Transplantation (PORT) Study. Am J Transpl. 2010; 10:338-353.

10. Kasiske BL, Guijarro C, Massy ZA, Wiederkehr MR, Ma JZ, et al. Cardiovascular disease after renal transplantation. J Am Soc Nephrol. 1996; 7:158-65. [PubMed: 8808124]

11. Kidney Disease Working Group. Kidney Disease: Improving Global Outcomes (KDIGO) 2012 clinical practice guideline for the evaluation and management of chronic kidney disease. Kidney Int. 2013; 3:1-150.

12. Gansevoort RT, Correa-Rotter R, Hemmelgarn BR, Jafar TH, Heerspink HJL, et al. Chronic kidney disease and cardiovascular risk: Epidemiology, mechanisms, and prevention. Lancet. 2013; 382:339-352. [PubMed: 23727170]

13. Matsushita K, Coresh J, Sang Y, Chalmers J, Fox C, et al. Estimated glomerular filtration rate and albuminuria for prediction of cardiovascular outcomes: A collaborative meta-analysis of individual participant data. Lancet Diabetes Endocrinol. 2015; 3:514-525. [PubMed: 26028594]

14. Matsushita K, van der Velde M, Astor BC, Woodward M, Levey AS, et al. Association of estimated glomerular filtration rate and albuminuria with all-cause and cardiovascular mortality in general population cohorts: a collaborative meta-analysis. Lancet. 2010; 375:2073-2081. [PubMed: 20483451]

15. Nitsch D, Grams M, Sang Y, Black C, Cirillo M, et al. Associations of estimated glomerular filtration rate and albuminuria with mortality and renal failure by sex: A meta-analysis. BMJ. 2013; 346:1-14.

16. Hippisley-Cox J, Coupland C, Brindle P. Development and validation of QRISK3 risk prediction algorithms to estimate future risk of cardiovascular disease: prospective cohort study. BMJ. 2017; 357:j2099. [PubMed: 28536104]

17. Piepoli MF, Hoes AW, Agewall S, Albus C, Brotons C, et al. 2016 European Guidelines on cardiovascular disease prevention in clinical practice. Eur Heart J. 2016; 37:2315-2381. [PubMed: 27222591]

18. Goff DC, Lloyd-Jones DM, Bennett G, Coady S, D’Agostino RB, et al. 2013 ACC/AHA guideline on the assessment of cardiovascular risk: A report of the American college of cardiology/American heart association task force on practice guidelines. Circulation. 2014:129.

19. Levey AAS, Stevens LA, Schmid CH, Zhang YY, Castro AAF 3rd, et al. A New Equation to Estimate Glomerular Filtration Rate. Ann Intern Med. 2009; 150:604-612. [PubMed: 19414839]

20. Inker LA, Schmid CH, Tighiouart H, Eckfeldt JH, Feldman HI, et al. Estimating glomerular filtration rate from serum creatinine and cystatin C. N Engl J Med. 2012; 367:20-9. [PubMed: 22762315]

21. Shlipak MG, Matsushita K, Ärnlöv J, Inker LA, Katz R, et al. Cystatin C versus Creatinine in Determining Risk Based on Kidney Function. N Engl J Med. 2013; 369:932-943. [PubMed: 24004120]

22. Shlipak MG, Mattes MD, Peralta CA. Update on cystatin C: Incorporation into clinical practice. Am J Kidney Dis. 2013; 62:595-603. [PubMed: 23701892]

23. National Institute for Health and Care Excellence \& NICE. Costing statement: Chronic kidney disease Implementing the NICE guideline on chronic kidney disease (CG182). 2014

24. NICE. Chronic kidney disease in adults : assessment and management. NICE Guidel. 2018

25. Myers GL, Miller WG, Coresh J, Fleming J, Greenberg N, et al. Recommendations for improving serum creatinine measurement: A report from the Laboratory Working Group of the National Kidney Disease Education Program. Clin Chem. 2006; 52:5-18. [PubMed: 16332993]

26. Zi M, Xu Y. Involvement of cystatin C in immunity and apoptosis. Immunol Lett. 2018; 196:8090. [PubMed: 29355583]

27. Salgado JV, Souza FL, Salgado BJ. How to understand the association between cystatin C levels and cardiovascular disease: Imbalance, counterbalance, or consequence? J Cardiol. 2013; 62:331335. [PubMed: 23849291]

28. Van Diepen M, Ramspek CL, Jager KJ, Zoccali C, Dekker FW. Prediction versus aetiology: Common pitfalls and how to avoid them. Nephrol Dial Transplant. 2017; 32:ii1-ii5. [PubMed: 28339854] 
29. Lamb EJ, Brettell EA, Cockwell P, Dalton N, Deeks JJ, et al. The eGFR-C study: accuracy of glomerular filtration rate (GFR) estimation using creatinine and cystatin $\mathrm{C}$ and albuminuria for monitoring disease progression in patients with stage 3 chronic kidney disease - prospective longitudinal study in a multiethnic. BMC Nephrol. 2014; 15

30. Tangri N, Stevens L, Griffith J, Tighiouart H, Djurdjev O, et al. A Predictive Model for Progression of Chronic Kidney Disease to Kidney Failure. Jama. 2011; 305:1553. [PubMed: 21482743]

31. Herrington WG, Emberson J, Mihaylova B, Blackwell L, Reith C, et al. Impact of renal function on the effects of LDL cholesterol lowering with statin-based regimens: a meta-analysis of individual participant data from 28 randomised trials. Lancet Diabetes Endocrinol. 2016; 4:829_ 839. [PubMed: 27477773]

32. Baigent C, Landray MJ, Reith C, Emberson J, Wheeler DC, et al. The effects of lowering LDL cholesterol with simvastatin plus ezetimibe in patients with chronic kidney disease (Study of Heart and Renal Protection): A randomised placebo-controlled trial. Lancet. 2011; 377:2181-2192. [PubMed: 21663949]

33. KDIGO. Clinical Practice Guideline for Lipid Management in Chronic Kidney DiseaseKidney Int. 2013; 3:182-189.

34. The SPRINT Research Group. A Randomized Trial of Intensive versus Standard Blood-Pressure ControlN Engl J Med. 2015; 373:2103-2116. [PubMed: 26551272]

35. Cheung AK, Rahman M, Reboussin DM, Craven TE, Greene T, et al. Effects of Intensive BP Control in CKD. J Am Soc Nephrol. 2017; 28:2812-2823. [PubMed: 28642330]

36. Welsh C, Celis-Morales CA, Brown R, Mackay DF, Lewsey J, et al. Comparison of Conventional Lipoprotein Tests and Apolipoproteins in the Prediction of Cardiovascular Disease: Data from UK Biobank. Circulation. 2019; doi: 10.1161/CIRCULATIONAHA.119.041149

37. Van Der Velde M, Matsushita K, Coresh J, Astor BC, Woodward M, et al. Lower estimated glomerular filtration rate and higher albuminuria are associated with all-cause and cardiovascular mortality. A collaborative meta-analysis of high-risk population cohorts. Kidney Int. 2011; 79:1341-1352. [PubMed: 21307840]

38. National Cardiovascular Intelligence Network, Public Health England. Chronic kidney disease prevalence model. 2014

39. Witte EC, Lambers Heerspink HJ, de Zeeuw D, Bakker SJL, de Jong PE, et al. First Morning Voids Are More Reliable Than Spot Urine Samples to Assess Microalbuminuria. J Am Soc Nephrol. 2009; 20:436-443. [PubMed: 19092125]

40. Elliott P, Peakman TC. The UK Biobank sample handling and storage protocol for the collection, processing and archiving of human blood and urine. Int J Epidemiol. 2008; 37:234-244. [PubMed: 18381398]

41. Fry A, Littlejohns TJ, Sudlow C, Doherty N, Adamska L, et al. Comparison of Sociodemographic and Health-Related Characteristics of UK Biobank Participants with Those of the General Population. Am J Epidemiol. 2017; 186:1026-1034. [PubMed: 28641372]

42. Byrne C, Caskey F, Dawnay CC, Ford D, Lambie FS, et al. UK Renal Registry UK Renal Registry 19th Annual Report of the Renal Association. Nephron. 2017:137.

43. Allen N, Sudlow C, Downey P, Peakman T, Danesh J, et al. UK Biobank: Current status and what it means for epidemiology. Heal Policy Technol. 2012; 1:123-126.

44. UK Biobank. UK Biobank: Protocol for a large-scale prospective epidemiological resource UK Biobank Coordinating Centre Stockport. 2007.

45. Bush K, Nolan J, Zhang Q, Herrington W, Sudlow C. Definitions of End Stage Renal Disease for UK Biobank Phase 1 Outcomes Adjudication Documentation prepared by: On behalf of UK Biobank Outcome Adjudication Group Definitions of End Stage Renal Disease, UK Biobank phase 1 outcomes adjudication. 2017

46. UK Biobank. [Accessed: 17th April 2019] UK Biobank Showcase. Blood Sample Collection, Processing and Transport. 2011. Available at: https://biobank.ctsu.ox.ac.uk/showcase/docs/ Bloodsample.pdf.

47. UK Biobank. [Accessed: 17th April 2019] UK Biobank showcase. Biospeciments manual: Collection of biological samples, processing and storage. 2011. Available at: https:// biobank.ctsu.ox.ac.uk/crystal/docs/BioSampleProc.pdf. 
48. UK Biobank. [Accessed: 17th April 2019] UK Biobank showcase. Companion Document to Accompany Serum Biomarker Data. Version 1.0. 2011. Available at: https://biobank.ctsu.ox.ac.uk/ showcase/docs/serum_biochemistry.pdf.

49. UK Biobank's Enhancements Working Group. [Accessed: 9th May 2019] UK Biobank biomarker panel; 2014. 1-4. Available at: http://www.ukbiobank.ac.uk/wp-content/uploads/2013/11/ ukb_biomarker_panel_final_website_May14.pdf.

50. UK Biobank. Details of assays and quality control information for the urinary biomarker data. v1.0. UK Biobank Showcase2016.

51. UK Biobank. Biomarker assay quality procedures: approaches used to minimise systematic and random errors. Version 2.0. UK Biobank showcase2019. Available at: https://

biobank.ctsu.ox.ac.uk/showcase/docs/biomarker_issues.pdf. [Accessed: 17th April 2019] 

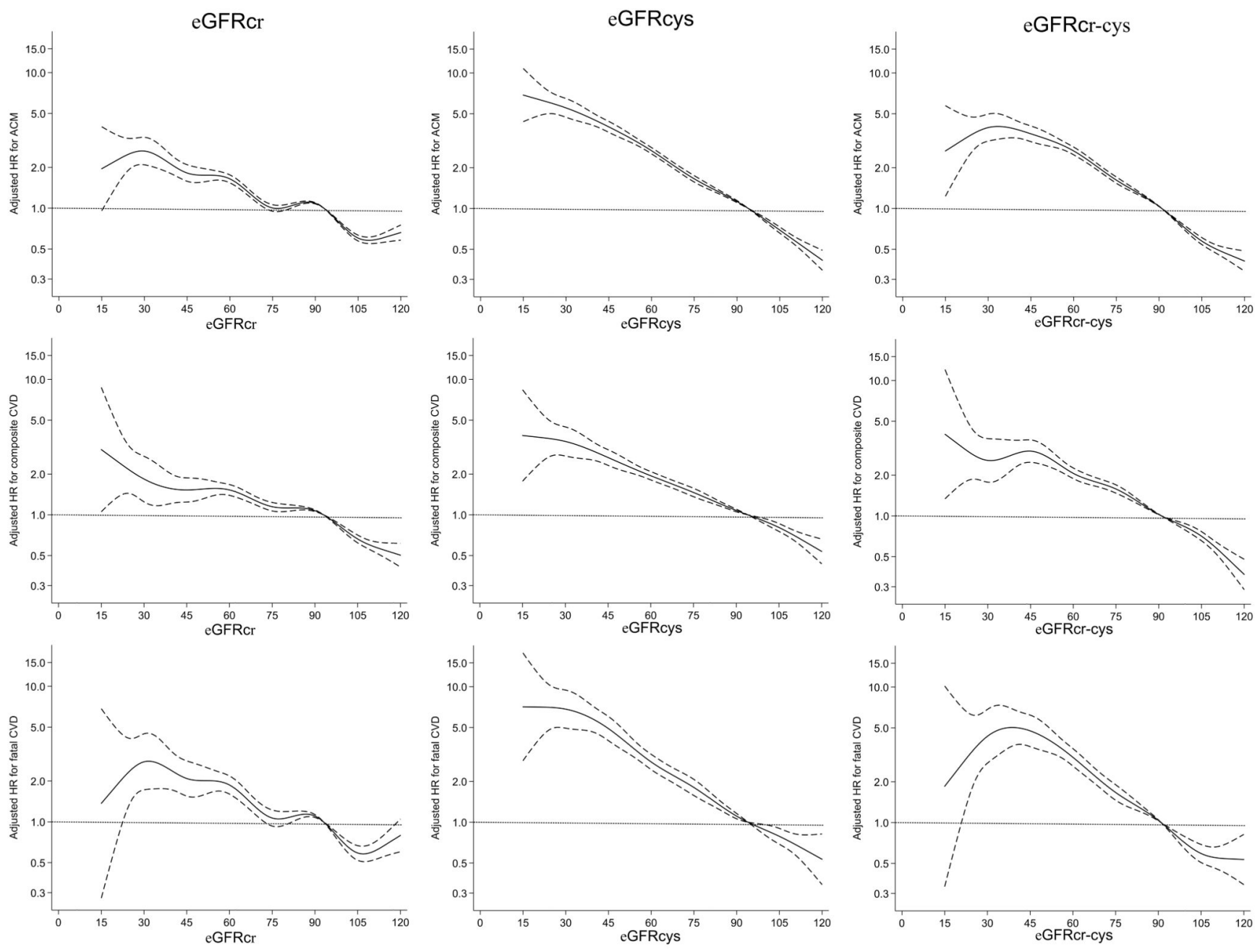

Figure 1.

Fully adjusted splines of estimated glomerular filtration rate (eGFR) against adjusted hazard ratio (with $95 \%$ confidence limits) for all-cause mortality (top row), composite fatal/nonfatal cardiovascular disease (CVD) (second row) and fatal CVD (third row) using eGFR based on creatinine (eGFRcr; left column), eGFR based on cystatin C (eGFRcys; middle column) and eGFR based on creatinine and cystatin C (eGFRcr-cys; right column). 


\begin{tabular}{|c|c|c|c|c|c|c|c|c|c|c|c|c|c|}
\hline & & \multicolumn{3}{|c|}{ UACR $(\mathrm{mg} / \mathrm{mmol})$} & \multicolumn{3}{|c|}{ uACR (mg/mmol) } & \multicolumn{3}{|c|}{ UACR $(\mathrm{mg} / \mathrm{mmol})$} & \multicolumn{3}{|c|}{ UACR $(\mathrm{mg} / \mathrm{mmol})$} \\
\hline \multirow{3}{*}{ eGFRcr } & & $<3$ & $3-30$ & $>30$ & $<3$ & $3-30$ & $>30$ & $<3$ & $3-30$ & $>30$ & $<3$ & $3-30$ & $>30$ \\
\hline & $\geq 90$ & 1 (ref) & no data & no data & 1 (ref) & no data & no data & 1 (ref) & no data & no data & 1 (ref) & no data & no data \\
\hline & $60-89$ & 0.92 & 1.11 & 0.90 & 1.06 & 1.04 & 1.12 & 0.98 & 1.15 & 0.95 & 1.13 & 5.35 & 3.63 \\
\hline \multirow{3}{*}{$\begin{array}{c}(\mathrm{ml} / \mathrm{min} / \\
\left.1.73 \mathrm{~m}^{2}\right)\end{array}$} & $45-59$ & 1.37 & 1.56 & 1.54 & 1.35 & 1.73 & 1.69 & 1.64 & 2.51 & 1.12 & 7.86 & 31.6 & 40.9 \\
\hline & $30-44$ & 1.78 & 2.59 & 1.72 & 1.53 & 1.61 & 2.38 & 1.66 & 3.38 & 2.42 & 43.8 & 245 & 210 \\
\hline & $15-30$ & 4.63 & 2.48 & 3.36 & 2.38 & 2.23 & 2.05 & 6.29 & 0.98 & 3.12 & 864 & 887 & 728 \\
\hline & & \multicolumn{3}{|c|}{ All-cause mortality } & \multicolumn{3}{|c|}{ Fatal/non-fatal CVD } & \multicolumn{3}{|c|}{ Fatal CVD } & \multicolumn{3}{|c|}{ End-stage kidney disease } \\
\hline & & \multicolumn{3}{|c|}{ uACR $(\mathrm{mg} / \mathrm{mmol})$} & \multicolumn{3}{|c|}{ uACR (mg/mmol) } & \multicolumn{3}{|c|}{ uACR $(\mathrm{mg} / \mathrm{mmol})$} & \multicolumn{3}{|c|}{ uACR $(\mathrm{mg} / \mathrm{mmol})$} \\
\hline \multirow{3}{*}{ eGFRcys } & & $<3$ & 3-30 & $>30$ & $<3$ & $3-30$ & $>30$ & $<3$ & $3-30$ & $>30$ & $<3$ & $3-30$ & $>30$ \\
\hline & $\geq 90$ & 1 (ref) & no data & no data & 1 (ref) & no data & no data & 1 (ref) & no data & no data & 1 (ref) & no data & no data \\
\hline & $60-89$ & 1.31 & 1.46 & 1.45 & 1.20 & 1.26 & 1.53 & 1.34 & 1.86 & 1.64 & 1.77 & 9.48 & 4.31 \\
\hline \multirow{6}{*}{$\begin{array}{c}(\mathrm{ml} / \mathrm{min} / \\
\left.1.73 \mathrm{~m}^{2}\right)\end{array}$} & $45-59$ & 2.19 & 2.44 & 2.96 & 1.69 & 1.92 & 2.19 & 2.65 & 2.47 & 3.82 & 6.96 & 70.1 & 40.0 \\
\hline & $30-44$ & 3.67 & 4.05 & 4.22 & 2.51 & 2.58 & 2.44 & 4.37 & 6.39 & 5.76 & 58.6 & 368 & 383 \\
\hline & $15-30$ & 5.36 & 7.10 & 6.76 & 4.35 & 2.68 & 5.30 & 8.51 & 7.91 & 11.2 & 580 & 2680 & 1726 \\
\hline & & \multicolumn{3}{|c|}{ All-cause mortality } & \multicolumn{3}{|c|}{ Fatal/non-fatal CVD } & \multicolumn{3}{|c|}{ Fatal CVD } & \multicolumn{3}{|c|}{ End-stage kidney disease } \\
\hline & & \multicolumn{3}{|c|}{ UACR $(\mathrm{mg} / \mathrm{mmol})$} & \multicolumn{3}{|c|}{ uACR $(\mathrm{mg} / \mathrm{mmol})$} & \multicolumn{3}{|c|}{ uACR $(\mathrm{mg} / \mathrm{mmol})$} & \multicolumn{3}{|c|}{ UACR $(\mathrm{mg} / \mathrm{mmol})$} \\
\hline & & $<3$ & $3-30$ & $>30$ & $<3$ & $3-30$ & $>30$ & $<3$ & $3-30$ & $>30$ & $<3$ & $3-30$ & $>30$ \\
\hline \multirow[b]{2}{*}{ eGFRcr-cys } & $\geq 90$ & 1 (ref) & no data & no data & 1 (ref) & no data & no data & 1 (ref) & no data & no data & 1 (ref) & no data & no data \\
\hline & $\overline{60-89}$ & 1.20 & 1.41 & 1.26 & 1.20 & 1.10 & 1.48 & 1.27 & 1.55 & 1.44 & 1.45 & 18.3 & 6.19 \\
\hline \multirow{4}{*}{$\begin{array}{c}(\mathrm{ml} / \mathrm{min} / \\
\left.1.73 \mathrm{~m}^{2}\right)\end{array}$} & $45-59$ & 2.16 & 2.53 & 2.25 & 1.83 & 2.08 & 2.16 & 2.70 & 3.43 & 3.43 & 13.7 & 264 & 163 \\
\hline & $30-44$ & 3.16 & 3.80 & 3.19 & 2.48 & 1.95 & 2.27 & 3.28 & 5.09 & 4.49 & 106 & 1454 & 887 \\
\hline & $15-30$ & 4.45 & 2.75 & 3.59 & 2.53 & 0.99 & 3.65 & 2.26 & 2.65 & 4.84 & 1023 & 10313 & 2945 \\
\hline & & \multicolumn{3}{|c|}{ All-cause mortality } & \multicolumn{3}{|c|}{ Fatal/non-fatal CVD } & \multicolumn{3}{|c|}{ Fatal CVD } & \multicolumn{3}{|c|}{ End-stage kidney disease } \\
\hline
\end{tabular}

Figure 2.

Heat maps for prediction of all-cause mortality, composite fatal/non-fatal cardiovascular disease (CVD), fatal CVD and end-stage kidney disease using eGFRcys and albuminuria (urine albumin:creatinine ratio; uACR) for estimated glomerular filtration rate (eGFR) based on creatinine (eGFRcr; top), eGFR based on cystatin C (eGFRcys; middle) and eGFR based on creatinine and cystatin C (eGFRcr-cys; bottom). No data were available for those with eGFR $>90 \mathrm{ml} / \mathrm{min} / 1.73 \mathrm{~m}^{2}$ and $\mathrm{uACR}>3 \mathrm{mg} / \mathrm{mmol}$. Otherwise, hazard ratios adjusted for atherosclerotic risk factors (age, sex, ethnicity, systolic and diastolic blood pressure, antihypertensive medications, statin use, smoking, diabetes, total and high-density lipoprotein cholesterol) were ranked 1-13 (1 being the lowest risk), and heat maps were colour-coded for all outcomes: 1-4 (green), 5-7 (yellow), 8-10 (orange), 11-13 (red). 


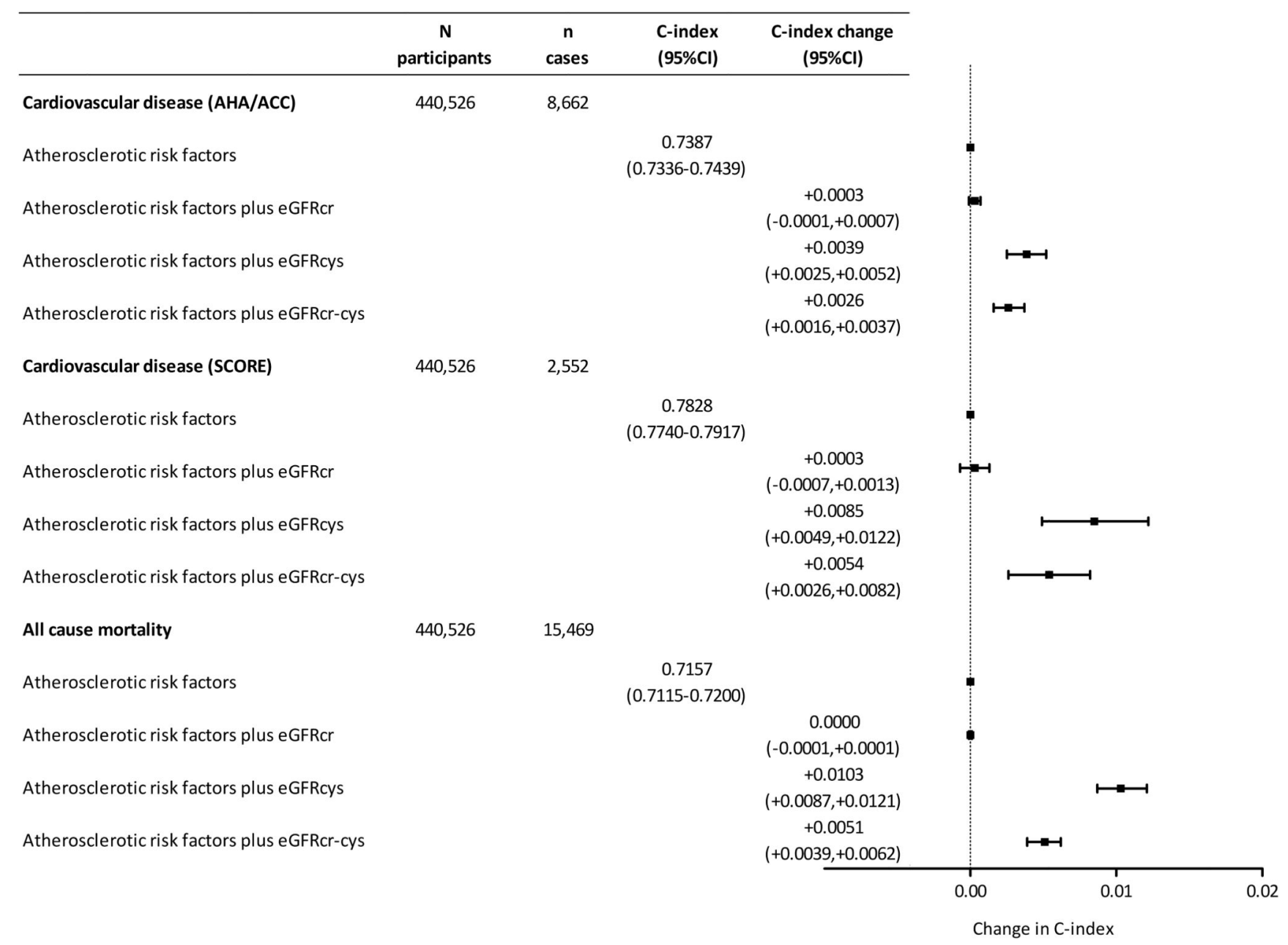

Figure 3.

Change in C-statistic with $95 \%$ confidence intervals for composite fatal/non-fatal cardiovascular disease, fatal cardiovascular disease or all-cause mortality upon addition of each estimated glomerular filtration rate (eGFR) method: eGFR based on creatinine (eGFRcr), eGFR based on cystatin C (eGFRcys) and eGFR based on creatinine and cystatin $\mathrm{C}$ (eGFRcr-cys). The centre line (0.00) represents no change to C-index. 


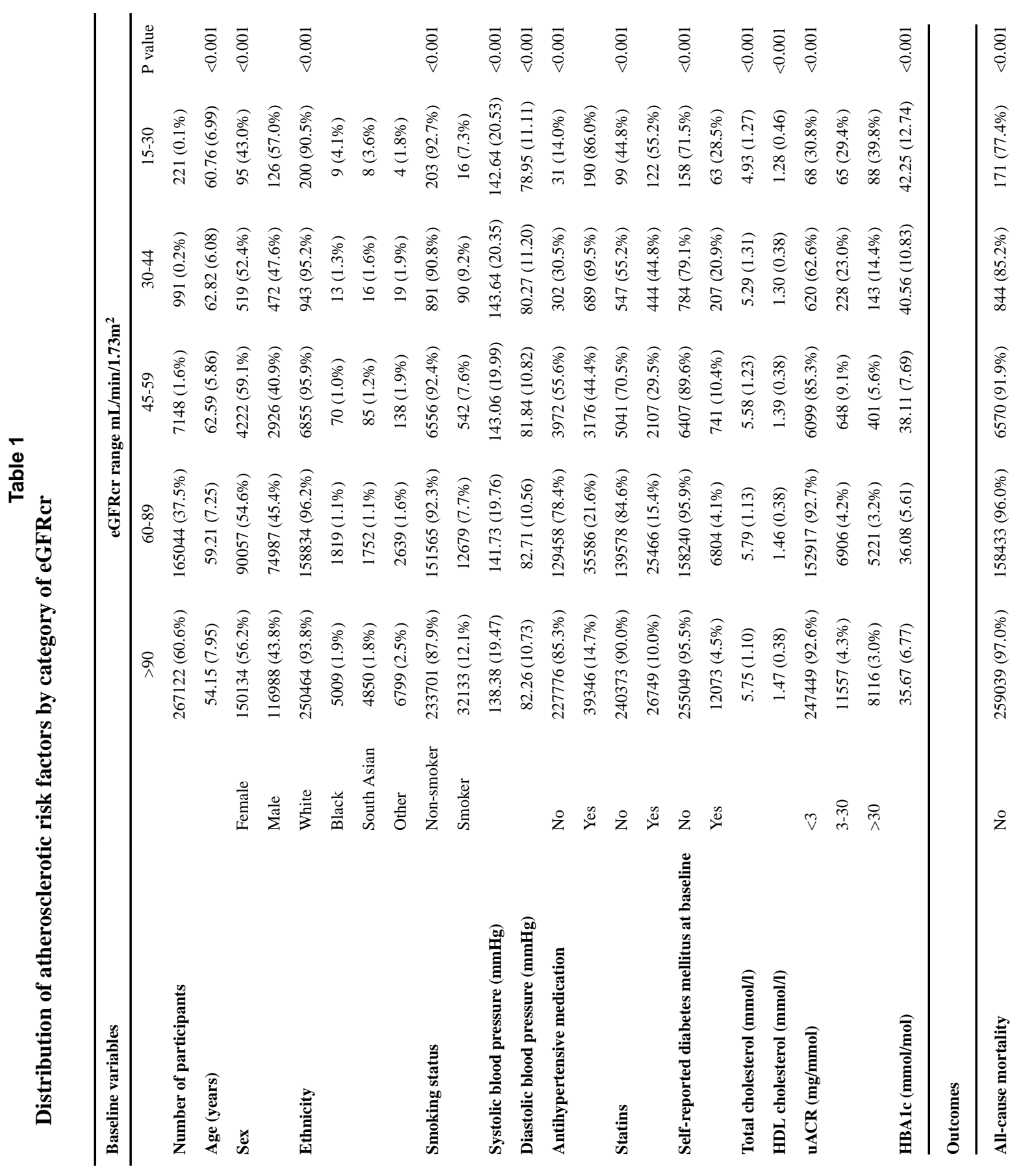




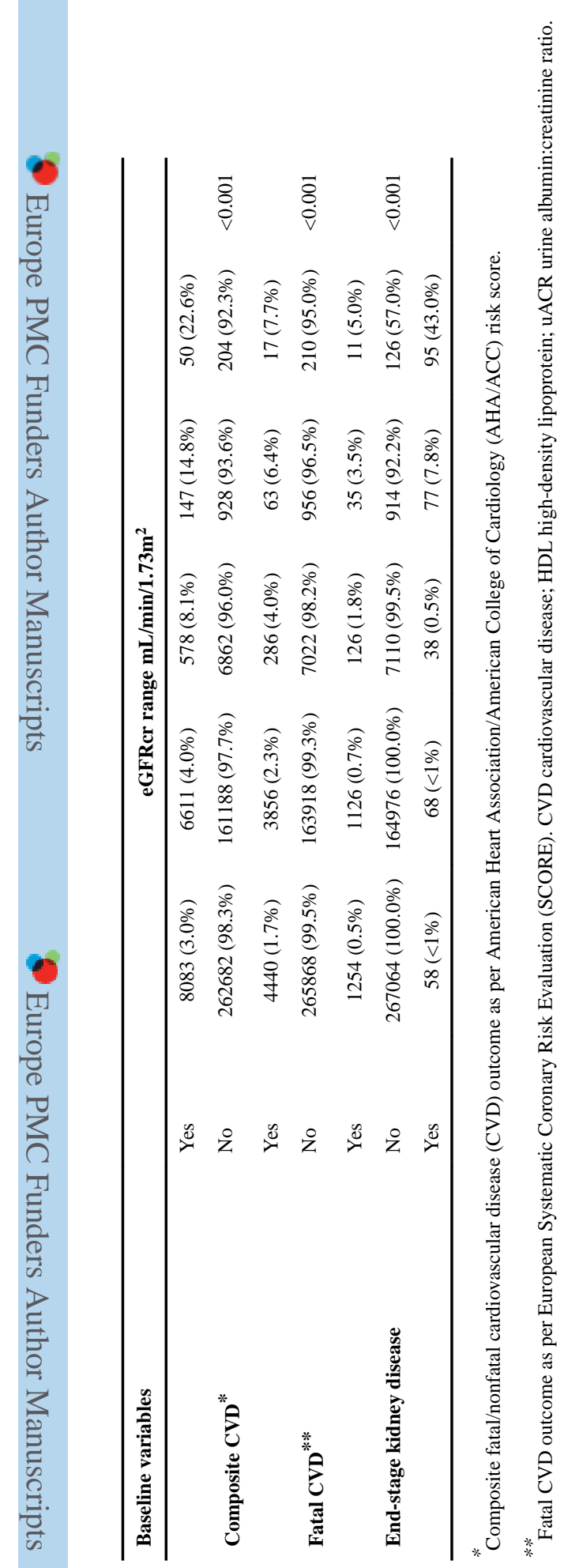


Table 2

Adjusted* hazard ratios of each eGFR measure (per $\left.10 \mathrm{~mL} / \mathrm{min} / 1.73 \mathrm{~m}^{2}\right)$ for each of the four outcomes, among 440,526 UK Biobank participants.

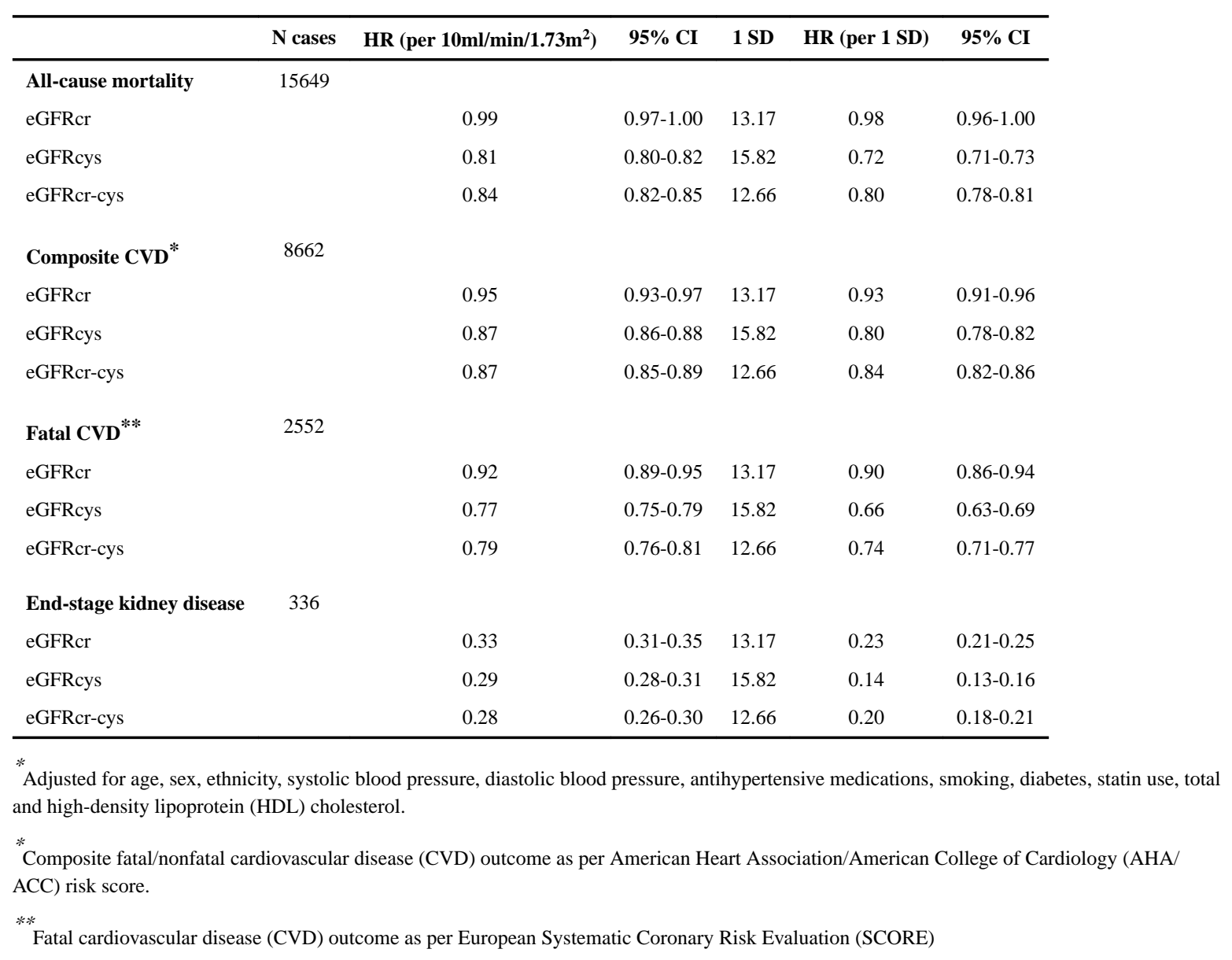


Table 3

Net Reclassification Index for composite fatal/non-fatal CVD outcome for 3 eGFR measures: eGFRcr, eGFRcys and eGFRcr-cys

\begin{tabular}{|c|c|c|c|}
\hline Comparator & Addition & $\begin{array}{l}\text { Case NRI } \\
(95 \% \mathrm{CI})\end{array}$ & $\begin{array}{c}\text { Non-case NRI } \\
(95 \% \text { CI })\end{array}$ \\
\hline Atherosclerotic risk factors & +eGFRcr & $\begin{array}{c}-0.08 \% \\
(-0.41,+0.22 \%)\end{array}$ & $\begin{array}{c}-0.01 \% \\
(-0.03,+0.01 \%)\end{array}$ \\
\hline Atherosclerotic risk factors & +eGFRcys & $\begin{array}{c}+1.54 \% \\
(+1.01,+2.10 \%)\end{array}$ & $\begin{array}{c}-0.17 \% \\
(-0.21,-0.14 \%)\end{array}$ \\
\hline Atherosclerotic risk factors & +eGFRcr-cys & $\begin{array}{c}+1.02 \% \\
(+0.53,+1.48 \%)\end{array}$ & $\begin{array}{c}-0.11 \% \\
(-0.14,-0.08 \%)\end{array}$ \\
\hline Atherosclerotic risk factors +eGFRcr & $+\mathrm{uACR}$ & $\begin{array}{c}0.08 \% \\
(-0.13,+0.19 \%)\end{array}$ & $\begin{array}{c}+0.01 \% \\
(0.00,+0.02 \%)\end{array}$ \\
\hline Atherosclerotic risk factors +eGFRcys & $+\mathrm{uACR}$ & $\begin{array}{c}-0.03 \% \\
(-0.15,+0.09 \%)\end{array}$ & $\begin{array}{c}+0.02 \% \\
(0.01,+0.03 \%)\end{array}$ \\
\hline Atherosclerotic risk factors +eGFRcr-cys & $+\mathrm{uACR}$ & $\begin{array}{c}0.07 \% \\
(-0.05,+0.18 \%)\end{array}$ & $\begin{array}{c}+0.01 \% \\
(0.00,+0.02 \%)\end{array}$ \\
\hline
\end{tabular}

Estimated glomerular filtration rate (eGFR) method: eGFR based on creatinine (eGFRcr), eGFR based on cystatin C (eGFRcys) and eGFR based on creatinine and cystatin C (eGFRcr-cys). uACR urine albumin:creatinine ratio 
Table 4

Change in Harrell's C-statistic for prediction of all-cause mortality, composite and fatal CVD outcomes and end-stage kidney disease in those with $\geq 20 \%$ absolute discordance between eGFRcr and eGFRcys

\begin{tabular}{lcccc}
\hline & N cases & C-statistic & Change in C-statistic & P value \\
\hline All-cause mortality & $6557 / 183867$ & $0.7207(0.7140-0.7275)$ & na & \\
+ eGFRcr & & $0.7270(0.7203-0.7337)$ & $0.0063(0.0042-0.0084)$ & $<0.001$ \\
+ eGFRcys & & $0.7343(0.7277-0.7410)$ & $0.0136(0.0108-0.0165)$ & $<0.001$ \\
+ eGFRcr-cys & & $0.7246(0.7178-0.7312)$ & $0.0038(0.0023-0.0054)$ & $<0.001$ \\
Composite CVD & $3565 / 183867$ & $0.7422(0.7338-0.7505)$ & na & \\
+ eGFRcr & & $0.7425(0.7341-0.7508)$ & $0.0003(-0.0003-0.0009)$ & 0.347 \\
+ eGFRcys & & $0.7469(0.7386-0.7552)$ & $0.0047(0.0027-0.0067)$ & $<0.001$ \\
+ eGFRcr-cys & & $0.7448(0.7365-0.7532)$ & $0.0027(0.0012-0.0042)$ & $<0.001$ \\
Fatal CVD & $1059 / 183867$ & $0.7947(0.7806-0.8088)$ & na & \\
+ eGFRcr & & $0.7965(0.7823-0.8107)$ & $0.0018(-0.0013-0.0049)$ & 0.264 \\
+ eGFRcys & & $0.8036(0.7897-0.8175)$ & $0.0089(0.0036-0.0141)$ & 0.001 \\
+ eGFRcr-cys & & $0.7979(0.7839-0.8119)$ & $0.0032(-0.0001-0.0065)$ & 0.057 \\
ESKD & $52 / 183867$ & $0.7200(0.6332-0.8068)$ & na & \\
+ eGFRcr & & $0.7215(0.6363-0.8067)$ & $0.0015(-0.0027-0.0058)$ & 0.483 \\
+ eGFRcys & & $0.7301(0.6378-0.8223)$ & $0.0101(-0.0473-0.0675)$ & 0.730 \\
+ eGFRcr-cys & & $0.7446(0.6649-0.8244)$ & $0.0246(-0.0337-0.0830)$ & 0.408 \\
\hline
\end{tabular}

C-statistics and change in C-statistic for Cox proportional hazard models adjusted for atherosclerotic risk factors (age, sex, ethnicity, smoking, systolic and diastolic blood pressure, antihypertensive medications, statins, total and high-density lipoprotein (HDL) cholesterol) and log urine albumin:creatinine ratio ( $\mathrm{UACR}$ ) with addition of the three estimated glomerular filtration rate (eGFR) methods: eGFR based on creatinine (eGFRcr), eGFR based on cystatin C (eGFRcys) and eGFR based on creatinine and cystatin C (eGFRcr-cys). 